\title{
Assessing the efficacy of two dual-active ingredients long-lasting insecticidal nets for the control of malaria transmitted by pyrethroid-resistant vectors in Benin: study protocol for a three-arm, single-blinded, parallel, cluster-randomized controlled trial
}

Manfred Accrombessi ${ }^{1 *}$ (D) Jackie Cook ${ }^{2}$, Corine Ngufor ${ }^{1}$, Arthur Sovi ${ }^{1,3,4}$, Edouard Dangbenon $^{3}$, Boulais Yovogan $^{3}$, Hilaire Akpovi ${ }^{3}$, Aurore Hounto ${ }^{5}$, Charles Thickstun ${ }^{6}$, Gil G. Padonou ${ }^{3}$, Filemon Tokponnon ${ }^{3}$, Louisa A. Messenger ${ }^{1}$, Immo Kleinschmidt ${ }^{2,7,8}$, Mark Rowland ${ }^{1}$, Martin C. Akogbeto ${ }^{3}$ and Natacha Protopopoff ${ }^{1}$

\begin{abstract}
Background: Long-lasting insecticidal nets (LLINS) are currently the primary method of malaria control in subSaharan Africa and have contributed to a significant reduction in malaria burden over the past 15 years. However, this progress is threatened by the wide-scale selection of insecticide-resistant malaria vectors. It is, therefore, important to accelerate the generation of evidence for new classes of LLINs.
\end{abstract}

Methods: This protocol presents a three-arm superiority, single-blinded, cluster randomized controlled trial to evaluate the impact of 2 novel dual-active ingredient LLINs on epidemiological and entomological outcomes in Benin, a malaria-endemic area with highly pyrethroid-resistant vector populations. The study arms consist of (i) Royal Guard ${ }^{\circledR}$ LLIN, a net combining a pyrethroid (alpha-cypermethrin) plus an insect growth regulator (pyriproxyfen), which in the adult female is known to disrupt reproduction and egg fertility; (ii) Interceptor G2 ${ }^{\circledast}$ LLIN, a net incorporating two adulticides (alpha-cypermethrin and chlorfenapyr) with different modes of action; and (iii) the control arm, Interceptor ${ }^{\circledR}$ LLIN, a pyrethroid (alpha-cypermethrin) only LLIN. In all arms, one net for every 2 people will be distributed to each household. Sixty clusters were identified and randomised 1:1:1 to each study arm. The primary outcome is malaria case incidence measured over 24 months through active case detection in a cohort of 25 children aged 6 months to 10 years, randomly selected from each cluster. Secondary outcomes include 1) malaria infection prevalence (all ages) and prevalence of moderate to severe anaemia in children under 5 years old, measured at 6 and 18 months postintervention; 2) entomological indices measured every 3 months using human landing catches over 24 months.

\footnotetext{
* Correspondence: manfred.accrombessi@lshtm.ac.uk

${ }^{1}$ Faculty of Infectious and Tropical Diseases, Disease Control Department, London School of Hygiene and Tropical Medicine, WC1E 7HT, London, UK Full list of author information is available at the end of the article
} 
(Continued from previous page)

Insecticide resistance intensity will also be monitored over the study period.

Discussion: This study is the second cluster randomised controlled trial to evaluate the efficacy of these nextgeneration LLINs to control malaria transmitted by insecticide-resistant mosquitoes. The results of this study will form part of the $\mathrm{WHO}$ evidence-based review to support potential public health recommendations of these nets and shape malaria control strategies of sub-Saharan Africa for the next decade.

Trial registration: ClinicalTrials.gov, NCT03931473, registered on 30 April 2019.

Keywords: Dual-active ingredient long-lasting insecticidal nets, Chlorfenapyr, pyriproxyfen, Royal Guard ${ }^{\circledR}$ Interceptor $^{\circledR}$ G2, Malaria case incidence, Malaria prevalence, Entomological inoculation rate, Cluster randomized controlled trial, Benin

\section{Background}

Insecticide-treated nets (ITNs) and, more recently, longlasting insecticidal nets (LLINs) are the most widely used preventive measure for controlling malaria in sub-Saharan Africa (SSA) [1]. The World Health Organization (WHO) estimates that over $50 \%$ of the SSA population now sleep under LLINs and together with improved diagnosis and treatment, nets have contributed to an estimated 42 and $66 \%$ reduction in malaria incidence and mortality, respectively, over the last 15 years [2]. Pyrethroids are currently the only type of insecticide used routinely on LLINs, and the rapid spread of pyrethroid-resistant vectors seriously threatens to reverse the gains achieved so far [3]. Indeed, several studies have demonstrated that LLINs are becoming less effective at killing mosquitoes in areas of high resistance compared to areas of susceptibility [4, 5], although epidemiological evidence remains inconclusive in some studies [6,7].

Due to concerns about the potential failure of current control tools as a result of insecticide resistance, WHO has encouraged manufacturers to develop new types of LLINs as part of the Global Plan for Insecticide Resistance Management in malaria vectors (GPRIM) [8]. The first nets to contain a mixture of active ingredients with evidence for impact on epidemiological outcomes were nets that combined a pyrethroid insecticide with the synergist piperonyl butoxide (PBO) which restores susceptibility to pyrethroid by neutralising mixed-function oxidase function responsible for resistance in vectors $[9,10]$.

New classes of ITNs combining two insecticides with differing modes of action could have the potential to improve vector control and delay the evolution of resistance and preserve the lifespan of both active ingredients (AI). The two most advanced products are the pyrethroid-pyriproxyfen LLIN (Olyset ${ }^{\odot}$ Duo and Royal Guard ${ }^{\circ}$ ) [11-14] and a pyrethroid-chlorfenapyr LLIN (Interceptor ${ }^{\circ}$ G2) [15-17]. Both types of LLINs have demonstrated improved efficacy in entomological studies but currently, limited epidemiological evidence has been generated. Most of the available evidence for these new nets are based on Phase I laboratory studies and Phase II experimental hut trials. Only Olyset ${ }^{\bullet}$ Duo has been tested in an randomized clinical trial (RCT) in Burkina Faso and demonstrated efficacy on malaria case reduction in comparison to clusters using standard pyrethroid nets [12].

There are currently no published data on the impact of Royal Guard ${ }^{\oplus}$ and Interceptor ${ }^{\circledR}$ G2 on malaria epidemiological outcomes. These LLINs need to be evaluated in the community to determine the full extent of their effectiveness for malaria control. Failure to do so will - as shown by the recent history of PBO LLINs $[18,19]$ - delay the scale-up of new and potentially effective tools due to lack of malaria control evidence. To receive a public health recommendation from WHO, new types of LLIN need to demonstrate improved epidemiological efficacy compared to standard pyrethroid LLIN in two settings [20]. A trial evaluating the same dual-AI LLINs is currently ongoing in Tanzania (clinical.gov, NCT03554616). West Africa constitutes a different environment and ecology from East Africa, with historically higher intensity of pyrethroid resistance in malaria vectors [21] and so far there is only limited entomological and epidemiological evidence that new mixture nets would have a better effect on malaria indicators compared to standard nets $[14,15]$.

The main objective of the present study is to assess the efficacy of two types of dual-AI LLINs on malaria in a setting where the main malaria vectors are resistant to pyrethroid insecticides. Secondary objectives are to assess the durability and bio-efficacy of the dual-AI LLINs in the community [22]; and to examine whether entomological outcomes from adapted experimental hut trials can predict epidemiological and transmission outcomes of the RCT using a malaria transmission model developed by Churcher et al. [23, 24]. The study protocol is reported in line with the Standard Protocol Items: Recommendations for Interventional Trials (SPIRIT) 2013 Statement [25].

\section{Study objectives \\ Epidemiology \\ Primary objective}

To evaluate the efficacy of two types of dual-AI LLINs compared to standard pyrethroid LLINs on malaria case 
incidence in children aged 6 months to 10 years during two years of follow up.

\section{Secondary objectives}

- To assess the efficacy of each dual-AI LLIN compared to a standard pyrethroid-only LLIN on malaria infection prevalence in all age groups, and prevalence of moderate to severe anaemia in children under 5 years old, at 6 and 18 months post-net distribution.

- To assess if dual-AI LLINs have a safety profile similar to pyrethroid-only LLINs in the population of the trial study area.

- To monitor and evaluate the equity of study nets coverage and usage in the trial.

\section{Entomology}

\section{Primary objective}

To assess the efficacy of each dual-AI LLINs compared to a standard pyrethroid-only LLIN on mosquito density, sporozoite rate, and the entomological inoculation rate (EIR) as a measure for malaria transmission.

\section{Secondary objectives}

- To assess the impact of the new type of LLINs on Anopheles vector survivorship and other entomological outcomes (e.g., mosquito resting behaviour, species composition, feeding, ovary development, and fecundity).

- To assess annual changes in insecticide resistance intensity to the three insecticides used on the nets.

\section{Methods/design}

\section{Study area}

The study site is situated in Cove, Zagnanado, and Ouinhi Districts, located in the Zou department, central Benin, $154 \mathrm{~km}$ north of Cotonou, the economic capital (Fig. 1). The area was selected because of (i) a prevalence of malaria infection between 20 to $40 \%$, (ii) high pyrethroid resistance in the main malaria vectors, (iii) and proximity to experimental hut sites. This area consists of 123 villages with approximately 54,000 households and population size of 220,000 inhabitants. The main economic activities of the population are farming, fishing, hunting, and trading $[26,27]$.

Malaria is highly endemic, and transmission occurs year-round. There are two rainy seasons, from April July and from October - November. Malaria infection prevalence in the Zou department was $20 \%$ in children under 5 years old according to the demographic health survey (DHS) conducted in 2011-2012 [28] and increased to $36.5 \%$ in the $2017-2018$ DHS [29]. The main vector control provision in the Zou department consists of the distribution of pyrethroid-only LLINs through regular universal coverage campaigns (most recently in 2017) and routine service delivery to pregnant women during antenatal care and to children under 5 years during extended programmes on immunization. According to the 2018 DHS, ITN usage in children under 5 years was $78.2 \%$.

The main vector species are Anopheles coluzzii and Anopheles gambiae sensu stricto. Entomological surveys performed in the Cove region in 2015 revealed high levels of pyrethroid resistance intensity ( $>200$ fold) mainly due to high frequencies of $\mathrm{kdr}$ of $>90 \%$ and elevated cytochrome P450 enzymes [30].

\section{Trial design}

The trial is a three-arm superiority, single-blinded, cluster-randomized trial with 20 clusters in each arm $(60$ clusters in total). The 3 arms are (i) mixture pyrethroid and chlorfenapyr LLIN: Interceptor ${ }^{\circ}$ G2 (Intervention 1), (ii) mixture pyrethroid-pyriproxyfen LLIN: Royal Guard ${ }^{\circ}$ (Intervention 2), (iii) pyrethroid-only standard LLIN: Interceptor ${ }^{\circ}$ (Control/reference arm). The study design is summarized in Fig. 2.

Each cluster will be comprised of 1 village or a group of villages for an average of 200 households (1200 residents) per cluster. Clusters will have a minimum of 100 children and 100 households. A "fried egg" design will be used for cluster boundaries. Clusters will be designed with core and buffer areas to reduce the likelihood of spill-over of intervention effects. Nets will be distributed to all households in a cluster (i.e. core and buffer areas) but data collection to measure intervention effects will be restricted to households situated in the core areas to avoid spillover from neighbouring clusters receiving different nets. The cluster demarcation will be performed using the spatial analyst toolbox in ArcGIS (ESRI, Redlands, USA) based on the following criteria; a minimum of 50 children aged 6 months to 10 years in the core area and a buffer of minimum $1000 \mathrm{~m}$ between core households and any households in an adjacent cluster.

To estimate malaria case incidence, a cohort of 25 children per cluster aged 6 months to 9 years will be randomly selected from a census list of children living in the study clusters and followed over 24 months. For malaria infection prevalence assessment, two cross-sectional surveys will be conducted at 6 and 18 months post-net distribution; 70 individuals per cluster will be randomly selected for each cross-sectional survey, stratified by age ( $<5$ years, $5-9$ years, $10-14$ years, $\geq 15$ years and older) from each of the 60 clusters.

Routine cross-sectional entomological surveys using human landing catches (HLCs) will be conducted in all clusters to assess the human biting rate and EIR. Each 


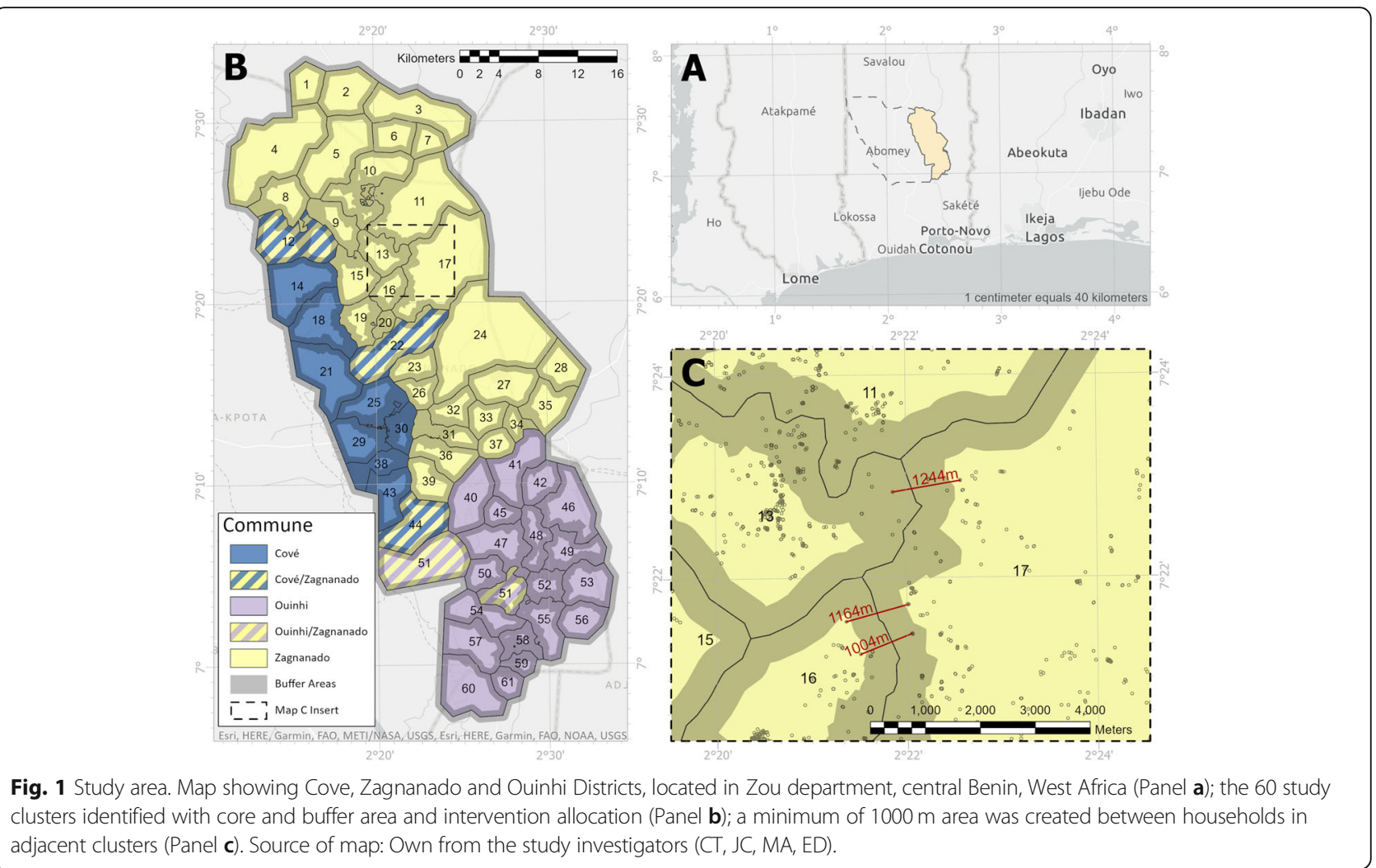

cluster will be visited once every three months. Four households will be selected from a census list in each study cluster at each time point. The first house will be randomly selected by the study data manager, while the other three will be chosen by the field team, in a radius of $15-20 \mathrm{~m}$ around the first one, to facilitate the supervision of mosquito collectors.

\section{Eligibility criteria}

The cohort will include children aged 6 months to 9 years old resident in the study villages, without any severe illnesses and whose parents/carers have given written informed consent for their child to be included in the study. Inclusion criteria during cross-sectional surveys will be the willingness to participate and provide

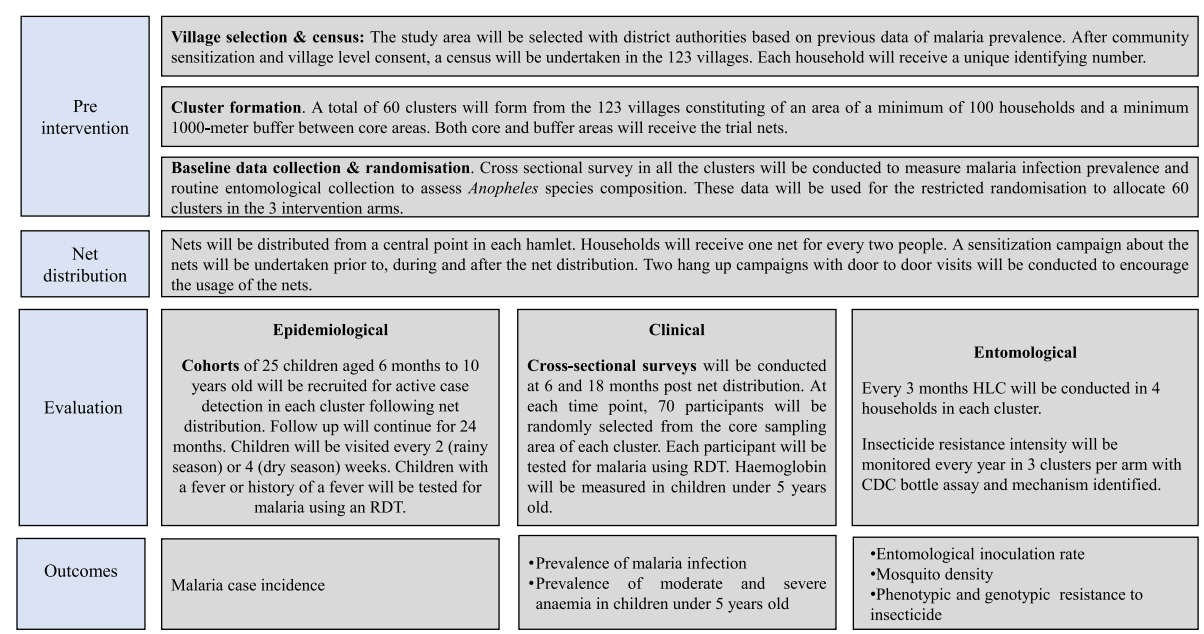

Fig. 2 Summary of study design 
consent (or for parents/carers to provide consent for children); resident in the village during the previous 3 months, selected individuals without severe illness.

\section{Interventions}

\section{Description of the study nets}

The nets are blue, rectangular, and identical sizes $(1.8 \mathrm{~m}$ long, $1.6 \mathrm{~m}$ wide, and $1.8 \mathrm{~m}$ high); however, the Royal Guard $^{\circ}$ textile is different from the other two nets (details below). The three net brands are all WHO prequalified vector control products [31].

Intervention 1: Interceptor ${ }^{\circ} \mathrm{G} 2$ (BASF Corporation) is a mixture LLIN made of polyester netting (100 deniers) coated with a wash-resistant formulation of $200 \mathrm{mg} / \mathrm{m}^{2}$ chlorfenapyr and $100 \mathrm{mg} / \mathrm{m}^{2}$ alpha-cypermethrin. Chlorfenapyr is a member of the pyrrole class of insecticides [32] and disrupts cellular respiration and oxidative phosphorylation in mitochondria and due to this unique mode of action is toxic to mosquitoes that are resistant to standard neurotoxic insecticides like pyrethroids [33]. No record of cross-resistance to chlorfenapyr has been reported up to now.

Intervention 2: Royal Guard ${ }^{\circ}$ (Disease Control Technologies, LLC) is a mixture LLIN made of polyethylene (120 deniers) incorporating $225 \mathrm{mg} / \mathrm{m}^{2}$ pyriproxyfen and $261 \mathrm{mg} / \mathrm{m}^{2}$ alpha-cypermethrin. Pyriproxyfen is a multiple acting compound (known as a juvenile hormone analogue) with insecticidal and sterilising effects on females which is known to disrupt reproduction and fertility of eggs [34].

Control: Interceptor ${ }^{\circ}$ LLIN (BASF Corporation) is a pyrethroid-treated LLIN with alpha-cypermethrin (coated onto filaments) at a target dose of $200 \mathrm{mg} / \mathrm{m}^{2}$ of polyester fabric (100 deniers). Interceptor was chosen as a direct comparison to Interceptor G2 and Royal Guard as all three nets are impregnated with alpha-cypermethrin pyrethroid (albeit at different concentrations) and because some pyrethroid resistance mechanisms involving CYP6 genes are specific to type I (permethrin) or type II (alphacypermethrin) pyrethroids [35].

\section{Intervention description}

Nets will be distributed with the support of the National Malaria Control Programme (NMCP). All households in the study area will receive one net for every two people. The census listing will be used to facilitate net distribution at a central location in each hamlet. To reach a minimum of $85 \%$ access following the distribution and a minimum of $75 \%$ usage, information, education, and communication (IEC) activities will be conducted before, during, and after LLIN distribution to increase usage in the study area, including instructions on when and how to wash the nets. A door to door hang-up campaign will take place after the distribution and depending on net usage rates, subsequent hang-up campaigns will be organized to increase net usage. Throughout the study, community health workers will be utilized to encourage continuous net use in the study population. Hamlet and religious leaders will be also involved in the sensitization campaigns for net usage.

The coverage achieved in each cluster will be evaluated through a post-intervention coverage survey one month after distribution. Net coverage and usage will also be assessed during cohort visits and cross-sectional surveys. Three indicators will be used: "proportion of households with at least one ITN for every 2 people", "proportion of household members with enough ITNs to sleep under (population access)" and "proportion of residents reporting using an ITN last night" [36, 37].

\section{Outcomes}

The primary outcome is malaria case incidence in children aged 6 months to 10 years (Table 1) over 24 months. A malaria case is defined as an infra-rouge frontal temperature above $37.5^{\circ} \mathrm{C}$ or history of a fever in the last $48 \mathrm{~h}$ and a positive rapid diagnostic test (RDT).

\section{Secondary outcomes are}

- Malaria infection prevalence in the study population at 6 and 18 months post bed net distribution,

- Prevalence of moderate to severe anaemia in children under 5 years old at 6 and 18 months post bed net distribution,

- EIR, as a measure for malaria transmission rate in the primary vector species.

\section{Other outcomes are:}

- Mosquito density and survivorship, mosquito resting behaviour, species composition, ovary development, and fecundity.

- Frequency and intensity of phenotypic and genotypic resistance to pyrethroid, chlorfenapyr, and pyriproxyfen insecticides.

- Prevalence of elevated cytochromeP450s and other metabolic enzymes associated with pyrethroid resistance [38].

\section{Sample size}

Sample size calculation for the primary outcome (malaria case incidence in children aged between 6 months and 10 years) was based on the method of Hayes and Bennett [39]. Based on passive data collected in 2018 by the NMCP in the study area, the mean number of malaria episodes per child per year in the reference arm was assumed to be 1 . We assumed a between-cluster coefficient variation of 0.3 and that dual-AI LLINs will reduce 
Table 1 Study outcomes, measurements, and process of collection

\begin{tabular}{|c|c|c|}
\hline Outcome & Measurement & Collection \\
\hline \multicolumn{3}{|l|}{ Epidemiological outcomes } \\
\hline Malaria case incidence & $\begin{array}{l}\text { Rapid diagnostic test taken when temperature } \geq 37.5^{\circ} \mathrm{C} \text { and/or history } \\
\text { of fever for the past } 48 \mathrm{~h}\end{array}$ & Active case detection: cohort follow-up \\
\hline Malaria infection prevalence & Rapid diagnostic test whatever the presence of malaria signs & Cross-sectional survey \\
\hline Moderate to severe anaemia & $\begin{array}{l}\text { Haemoglobin (measured by Haemocue), defined as }<10 \mathrm{~g} / \mathrm{dL} \text { and }<8 \mathrm{~g} / \\
\mathrm{dL} \text {, respectively. }\end{array}$ & $\begin{array}{l}\text { Active case detection: cohort follow-up } \\
\text { Cross-sectional survey }\end{array}$ \\
\hline Temperature & $\begin{array}{l}\text { Digital infra-red ear thermometer } \\
\text { Temperature and history of fever }\end{array}$ & $\begin{array}{l}\text { Active case detection: cohort follow-up } \\
\text { Cross-sectional survey }\end{array}$ \\
\hline \multicolumn{3}{|l|}{ Entomological outcomes } \\
\hline $\begin{array}{l}\text { Indoor and outdoor } \\
\text { Anopheles biting density }\end{array}$ & Human landing catch & Entomology monitoring \\
\hline Mosquito sporozoite rate & $\begin{array}{l}\text { Standard CSP-ELISA uses to estimate the EIR. The positive sample will be } \\
\text { confirmed with a second ELISA using a heating technique }\end{array}$ & Entomology monitoring \\
\hline $\begin{array}{l}\text { Anopheles species } \\
\text { identification }\end{array}$ & An. gambiae, An. coluzzii and An. funestus Taq Man Real time PCR & $\begin{array}{l}\text { Entomology monitoring } \\
\text { Sentinel site and resistance test }\end{array}$ \\
\hline Resistance intensity & WHO cylinder assay, collection of adult Anopheles resting indoor & Entomology monitoring \\
\hline Frequency of Vgsc mutation & Taq Man PCR & Entomology monitoring \\
\hline $\begin{array}{l}\text { Identification and intensity of } \\
\text { overexpressed CYP6 genes }\end{array}$ & $\begin{array}{l}\text { Screening for CYP6 genes by using Microarrays } \\
\text { Reverse-transcription quantitative PCR (RT-qPCR) using for confirming } \\
\text { and monitoring the expression of the cytochrome identified }\end{array}$ & $\begin{array}{l}\text { Resistance activity, unfed } 3 \text { days old } \\
\text { adult Anopheles from larval collection }\end{array}$ \\
\hline
\end{tabular}

Abbreviations: EIR Entomological inoculation rate

malaria case incidence by $30 \%$ (i.e. to 0.7 cases per child per year). With a follow-up of 2 years, to detect this effect size with $80 \%$ power would require following 20 children and 5 additional children to account for the loss of follow-up (25 in total) in 20 clusters in each of the three arms. This sample size calculation includes adjustment for multiple testing (allowing for the $3 \mathrm{arms}$ ) using a Bonferroni-corrected two-sided alpha of $1.67 \%$. This results in a total of 60 clusters required for the trial and a cohort of 1500 children.

For the cross-sectional surveys, it was assumed that malaria prevalence in the reference arm is $40 \%$, with a coefficient of variation between clusters of 0.3 . With 70 individuals per cluster and 20 clusters per arm, the study will have $80 \%$ power to detect a relative $30 \%$ lower prevalence (prevalence ratio 0.70) between each intervention arm to standard LLIN using a Bonferronicorrected two-sided alpha of $1.67 \%$ to account for multiple comparisons.

To achieve adequate participant enrolment to reach the target sample size, enhanced community sensitization activities will be conducted before and during each activity in the community with the support of hamlet leaders and community health workers.

\section{Assignment of interventions: allocation and blinding}

Restricted randomisation will be used to allocate the 60 clusters into the three study arms. Arms will be balanced on the following criteria: population size of the cluster (required to balance net numbers), infection prevalence, and socio-economic status. Data on these variables by cluster will be obtained through the pre-trial (baseline) cross-sectional survey.

The study is a single-blinded trial. Study participants will be blinded to the type of nets they have received. All field staff will be blinded to the allocation and analyses will be conducted on blinded data.

\section{Data collection, management, and analysis Census}

A short questionnaire will be used to collect demographic details on household residents to estimate the number of nets required to be distributed in each house and to randomly select children for the cohort. Each building will be mapped using a Global Positioning System to assist with delineating clusters.

\section{Cohort monitoring}

All children included in the cohort will be cleared of infection at enrolment using directly observed treatment with artemisinin-based combination therapy (ACT). During follow-up, visits will take place every two weeks during the malaria transmission season (from April to November) and every month during the dry season (from December to March). During each visit, children with fever (temperature $\geq 37.5^{\circ} \mathrm{C}$ ) or reporting a fever in the past $48 \mathrm{~h}$ will be tested for the presence of malaria parasites using RDT (SD Bioline Malaria Ag P.f, HRP2, Standard Diagnostics, Germany). If a child has a positive 
RDT, they will be recorded as a malaria case and will receive treatment as per national guidelines [40].

During the enrolment visit, a detailed questionnaire will be administered, to collect information on net ownership, household assets (as a proxy for socioeconomic status) and housing materials and window screening, and a clinical examination of the child will be conducted. In follow-up visits, a short questionnaire will be administered to record information on LLIN usage the night before, any adverse events encountered, and travel history. At each visit, children will also be examined by study nurses for signs of other illnesses and will be treated if needed. A child will be considered "lost to follow-up" if they miss at least 4 consecutive visits (i.e. 8 weeks of follow-up time).

\section{Cross-sectional surveys}

Each survey will include two components; i) a household survey and ii) a clinical survey. During the household survey, a questionnaire will be administered to obtain information on demographic and socioeconomic data, educational status, wealth assets of the household, vector control measures, and randomly sampled persons (2 per household) will be tested for malaria using an RDT, regardless of symptoms. Haemoglobin level will be measured with an HemoCue device (HemoCue Hb 201+, Aktiebolaget Leo Diagnostics, USA) in children under 5 who will receive iron tablets if they are found to be anaemic. Information will be collected on the perception and acceptance of nets. The occurrence of adverse events will also be reported.

\section{Entomology activities}

Entomological monitoring will take place every 3 months in each of the 60 study clusters. A standardized questionnaire will be used in each selected household to record the number of inhabitants, type of house (wall, roof, number of rooms, number of sleeping places), presence of animals, and malaria prevention measures used by household members. Mosquito collections will take place using HLCs. Four trained volunteer mosquito collectors from each study cluster will participate in house collections; one will be seated indoors and the second outdoors, and both will be replaced after $6 \mathrm{~h}$ of collection by the other two collectors. The collectors will be supervised by a team leader during the collection night.

Mosquitoes collected will be identified by microscopy and the numbers of An. gambiae s. l., An. funestus s. 1. and other anophelines recorded [41]. Human biting rate (outdoor and indoor) will be compared between study arms. Parity rates will be estimated in a subset of live Anopheles mosquitoes through dissection of ovaries [42]. The presence of Plasmodium falciparum circumsporozoite protein (Pf-CSP) in Anopheles vectors will be identified in a sub-sample (minimum of 50 Anopheles from indoor and outdoor collections per house) using an enzyme-linked immunosorbent assay (ELISA) [43]. All An. gambiae s.l. positive for Pf-CSP and a sub-sample of negative specimens will be identified to species-level by PCR [44]. The EIR will be calculated as the aggregated mean of sporozoite infective bites/person/year. In order to assess the impact of the pyrethroid-pyriproxyfen LLIN (Royal Guard ${ }^{\circledR}$ ) on mosquito reproduction, blood-fed Anopheles will be collected and ovary development recorded each year in houses from study arms, by dissection.

Insecticide resistance will be monitored annually using standard CDC intensity bottle assays [45] in the three arms to determine the prevalence of any selection of resistance to pyriproxyfen or chlorfenapyr, or change in resistance to pyrethroids. To monitor changes in insecticide resistance, adult Anopheles will be tested in CDC bottle bioassays using discriminating dosages of alphacypermethrin $(12.5 \mu \mathrm{g} / \mathrm{ml})$, chlorfenapyr $(100 \mu \mathrm{g} / \mathrm{ml})$ and pyriproxyfen $(100 \mu \mathrm{g} / \mathrm{ml})$ [46]; where mosquito mortality is $<90 \%$ to alpha-cypermethrin, intensity assays, testing two, five and ten times the discriminating dose will be performed. The percentage mortality at $30 \mathrm{~min}$ and 24 , 48 , and $72 \mathrm{~h}$ post-exposure will be recorded. Mosquitoes exposed to pyriproxyfen will be dissected to assess ovary development [47]. Tests will be performed at baseline and once per year post-intervention in two clusters per study arm.

All knockdown/dead mosquitoes after insecticidal exposure and surviving $72 \mathrm{~h}$ post-exposure to insecticides will be stored individually in RNAlater ${ }^{\bullet}$ and preserved at $-20{ }^{\circ} \mathrm{C}$ for gene expression analysis. Following species identification by PCR, RNA will be extracted from pools of confirmed An. gambiae s.s. or An. coluzzii and cDNA synthesized, according to standard procedures. Relative expression of CYPs and other metabolic enzymes, previously identified as being over-expressed in resistant $A n$. gambiae s.l. will be measured using multiplex TaqMan RT-qPCR assays [48].

\section{Data management}

Household data, clinical measurements in the cohort study, and entomological data collected during the cross-sectional surveys will be captured in electronic forms on smartphones installed with Open Data Kit (ODK) collect. The data will be stored on a secure server located at the London School of Hygiene and Tropical Medicine (LSHTM) and all data management and analyses will be done using Stata software.

\section{Data quality and control (QC)}

Standard operating procedures (SOPs) for data collection will be developed and field study staff will be 
appropriately trained to ensure rigorous data collection. QC will be conducted by a supervisor who will monitor the performance of field staff by checking for completeness and internal consistency of responses on the same day as data collection. Data collected on paper forms (mosquito identification, insecticide resistance results) will be double entered into a database independently by two data clerks. During the study, the data manager will prepare regular QC reports to document the status of data entry and any corrective actions needed.

Data security All data will be uploaded into a secure server on the LSHTM cloud. Data will be stored encrypted and will be accessible only by secure encryption keys and passwords. Access to the data will be restricted only to authorised study investigators and data management staff. A unique identifier number will be given to each participant and household to safeguard confidentiality.

Data storage Upon completion of the study, electronic files will be stored on a server and also copied to encrypted USB and stored offsite in a safe box. Case report forms (CRFs) will be stored in the secure archive equipped with locked cabinets for long-term storage. Electronic data and paper source records will be retained for a minimum of 10 years following the study completion.

\section{Statistical methods}

Descriptive statistics will be used to compare the characteristics of participants between the study arms. All primary analyses will be conducted by intention to treat and adjusted for the restriction variables used for the constrained randomisation.

Primary outcome To assess whether the new dual-AI LLINs are superior to the reference LLIN, malaria case incidence in each intervention arm (Royal Guard and Interceptor G2) will be compared to malaria case incidence in the reference arm (Interceptor). To adjust for the increased risk of type I error due to multiple pairwise comparisons, the level of significance will be adjusted using the Bonferroni method. Following any treatment for malaria, a child will not be considered at risk for two weeks. Multilevel mixed models with random effects will be used to test the difference in incidence rate between each intervention and the control arm, allowing for repeated measurements on the same individual, and within-cluster correlation of responses. Survival analyses will be also used to compare time to the first infection case, adjusting for confounding factors using a Cox proportional hazards model. Multiple events will be allowed per child (with appropriate censoring to account for prophylactic protection following treatment and absence during follow-up visits).

Secondary outcomes Prevalence of malaria infection in the study clusters will be estimated at baseline, 6 months, and 18 months. The surveys will be analysed separately and in a combined model, controlling for the time in the analyses. All analyses will account for the multilevel nature of the data using random-effects regression models. The prevalence of moderate and severe anaemia in children under 5 will be analysed using logistic regression.

EIR will be estimated as the mean number of sporozoite infected Anopheles (An. gambiae s.I.) collected in the HLCs per house per night and weighted to account for the proportion of collected Anopheles processed for sporozoites. Differences in Anopheles density and EIR between the different arms will be estimated using random-effects negative binomial regression taking into account the intra-cluster correlation and any confounding factors that are found to be imbalanced between arms. Random effects logistic regression will be used to compare sporozoite rates between study arms allowing for within cluster correlation of responses.

\section{Oversight and monitoring}

A Trial Steering Committee (TSC) and Data Safety and Monitoring Committee (DSMC) will be established to provide oversight for the study. The TSC and DSMC members will be independent of the trial and its institutions and have the necessary expertise to monitor study progress and participant safety. The DSMC will be responsible for monitoring the progress of the trial, adherence to the protocol, the safety data, and the critical efficacy endpoints.

The management of the trial is the responsibility of the chief investigator and co-investigators. They will ensure that data are recorded in compliance with good clinical practice, and all regulatory and institutional requirements.

Although the risk to study participants is considered minimal from any of the study LLINs, their safety profile will be documented. Any adverse events (AE) which have been reported to be associated with exposure to insecticides such as skin rashes, skin burning, skin itching, skin paraesthesia, watering eyes, runny nose, sneezing, mucosal irritation, headache dizziness, and their severity will be actively and passively monitored in the study cohort and study participants selected during the crosssectional survey. Serious adverse events (SAE) will be reported to DSMC and ethical committees. Field workers will be trained to handle any disclosure of personal information or health conditions reported sensitively and confidentially. 


\section{Ethics and dissemination \\ Research ethics approval}

This study has been approved by the ethical review committee of the Ministry of Health in Benin $\left(\mathrm{N}^{\circ} 6 / 30 / \mathrm{MS} /\right.$ DC/DRFMT/CNERS/SA), the institutional review board of London School of Hygiene and Tropical Medicine $\left(\mathrm{N}^{\circ} 16,237\right)$, and the WHO Research Ethics Review Committee (ERC.0003153). Any amendments to the protocol and informed consent forms will be submitted for approval to all three ethics committees. The trial is registered on clinicaltrials.gov (NCT03931473, registered on 30 April 2019).

The study will be conducted according to the Declaration of Helsinki and the International Guidelines for Ethical Review of Epidemiological Studies [49]. All field and clinical staff as well as the investigators will receive training on good clinical and laboratory practice before data collection starts.

\section{Informed consent procedure}

Before any project activities, villages and hamlet leaders and local health staff will be invited to sensitisation sessions. Community health workers within each cluster will be fully informed as to the aims of the trial and will be on hand to answer day to day questions regarding the study.

For all activities involving a household, written informed consent will be obtained from an adult guardian in the household or be given by the participant if over 18 years. The consent form will be in French and indicate the purpose of the study, procedures, risks, and benefits. All participation is completely voluntary, and participants can withdraw at any time. In case the participant does not understand French, study investigators will be trained to practice oral translation of the consent into local languages. Participants will be asked to sign the consent in duplicate, one will be kept by the project and the other will remain with the household. If the person consenting is unable to read or write, their fingerprint will be taken, and an impartial witness to the informed consent procedures signature will be requested to sign. For the active follow-up cohort, consent will be sought once at the first enrolment visit and will be performed by the study nurses. Assent will be sought for children over 10 years.

For HLCs, written informed consent will also be obtained from volunteer mosquito collectors before being involved in the study. Mosquito collectors will be over the age of 18 years. To prevent yellow fever, they will be vaccinated. Their health will be monitored by the project clinical team and free treatment for malaria will be provided, if necessary. Each volunteers will work one night every 3 months to reduce the risk.

\section{Confidentiality}

All procedures for data collection, management, storage, and manipulation will follow SOPs. To ensure confidentiality is maintained, paper CRFs with participants' names will be stored in a locked cabinet and only accessible by authorised staff. All analyses will be done using the unique identifier.

\section{Dissemination activities}

All findings of the trial will be shared with international policymakers such as the WHO Vector Control Advisory Group (VCAG), the malaria policy advisory group (MPAC), and the WHO prequalification team for vector control product for revision of WHO guidance on dualAI LLINs. The project will involve the engagement of national, local, and community authorities and leaders. Mid- and end of project meetings will be organized to share the progress with village and community leaders to help disseminate findings to the community.

\section{Discussion}

A new generation of ITNs is urgently needed to maintain and ideally improve on the gains achieved by standard pyrethroid LLINs over the past two decades [18] and to control malaria transmitted by pyrethroid-resistant vectors. There is still only minimal evidence for the use of these nets in areas of insecticide resistance $[9,10,12]$, and more evidence is required for consolidating the findings of these initial trials.

The most advanced dual-AI LLIN assessed until now are nets combining a synergist $\mathrm{PBO}$ and pyrethroid insecticide. Two large RCTs conducted in Tanzania [9] and Uganda [10] have shown better efficacy than standard LLINs on malaria infection prevalence. This has led to a recommendation from WHO that PBO nets can be deployed across SSA in areas where vectors are resistant to pyrethroid insecticides [50].

Pyriproxyfen-pyrethroids LLINs have shown improved efficacy and wash resistance relative to pyrethroid-treated nets in terms of mosquito mortality and prevention of blood-feeding in experimental hut trials in Benin [11]. Several phase II studies conducted in West Africa have also shown that this type of dual-AI LLIN had a significant impact on mosquitoes' fertility by sterilising a large proportion of surviving blood-fed female mosquitoes through the pyriproxyfen component $[13,14,51]$. To date, there is only one RCT that assessed pyriproxyfen-pyrethroid LLINs (Olyset ${ }^{\oplus}$ Duo). The trial was conducted in Burkina Faso over 18 months and showed $12 \%$ protective efficacy compared to the standard net on malaria case incidence but no reduction in infection prevalence [12]. The relatively small protective effect could be due to the short residual bio-efficacy of pyriproxyfen on the net. Indeed, the study investigators reported that fecundity indicators were 
only reduced in the first month after the use of the pyriproxyfen-pyrethroid LLINs, but not thereafter [52]. Royal Guard ${ }^{\circledR}$, also a dual pyriproxyfen-pyrethroid LLIN, [14] induced greater levels of suppression of mosquito reproduction in Phase I and II trials than that which was reported in similar studies with Olyset ${ }^{\circ}$ Duo [11]. Chlorfenapyr-pyrethroid LLINs have demonstrated better efficacy and wash resistance against pyrethroid-resistant mosquitos in comparison to standard alpha-cypermethrin LLINs in Phase I and II trials [15-17].

Currently, there is less evidence for the performance of dual-AI LLINs on epidemiological outcomes in West African communities where vectors usually display much higher levels of insecticide resistance intensity than those observed in East Africa, raising the concern that they might not be as effective in areas with intense insecticide resistance. The present trial will therefore answer a critical question about the efficacy of the most promising generation of LLINs in West Africa (except for PBOpyrethroid LLINs which were not included). It is the only clinical trial in the area to assess mixtures of adulticides with different modes of action (chlorfenapyr and pyriproxyfen) with alpha-cypermethrin. The findings of this trial together with those of a similar RCT conducted in Tanzania will be reviewed by the WHO VCAG and will contribute to the body of evidence to enable policy recommendations. If these two first-in-class products demonstrate superior efficacy to standard LLINs, they will be the first recommended LLINs impregnated with an insecticide class other than pyrethroids and $\mathrm{PBO}$ in the last three decades and will pave the way for the development of further LLINs. Any dual-AI LLIN that will be developed subsequently and share the same mode of actions would need to show non-inferiority against entomological outcomes in Phase II experimental hut conditions to be prequalified by WHO [53].

LLINs combining two insecticides of unrelated classes may become a key component of future insecticide resistance management by decreasing selection pressure (genotypic and phenotypic) among vector populations. While resistance monitoring in the proposed study is not powered to be able to detect significant changes, the trialling side by side of dual-AI LLINs and standard LLINs may allow us to detect important trends. Furthermore, profiling of insecticide resistance in the local vector will provide valuable information on the condition of deployment of these tools.

Both nets being tested contain a pyrethroid. The main effect of pyrethroids is to enhance the barrier offered by the netting material by killing and repelling mosquitoes and reducing blood feeding. The second AI in the dual LLINs cannot provide this level of individual protection. Neither chlorfenapyr nor pyriproxyfen has repellent effects. The main impact of the partner AIs will be to kill or sterilise mosquitoes that come into contact with the insecticides, therefore reducing the overall mosquito population. For both nets, the impact of these interventions is expected to be maximal at the community level when net usage is high. It will be therefore critical to achieve high population coverage. Several enhanced community sensitization activities and hang-up campaigns will be organized before, during, and after the LLIN distribution.

Nets have been one of the mainstays of vector control for several decades, primarily due to their ease of use and effectiveness. However, as anecdotal reports of their reduced efficacy are reported $[4,5,54]$, alongside evidence that new nets provide better protection, there is a danger that community willingness to use nets may wane. It is vital that confidence is maintained in these life-saving tools. If these new generations of LLINs are effective at reducing malaria, this trial will provide vital evidence to policy-makers and will enable stakeholders to consider these dual-AI LLINs as alternative solutions for malaria control in areas of insecticide resistance.

\section{List of protocol version}

Protocol version 4.0: 30/06/2019

\section{Trial status}

Baseline surveys are completed, and the cohort recruitment is currently running. Due to the COVID-19 pandemic and the cases confirmed in Benin, the recruitment of cohort children has been postponed from April to July 2020. Entomological collection using human landing catches were also suspended and resumed at the end of June. Laboratory work and hut trial work continued as normal. This delay has led to the loss of 3 months of data collection; therefore, the sample size has been modified accordingly. With a follow-up of 21 months, 30 children instead of 25 per cluster would be required for detecting the impact of the study nets. For mosquito collection, each cluster will be visited seven times over the 21 months follow-up period. The amendments have been submitted to the different ethics committee. All staff is using appropriate protective equipment and hand sanitation measures.

\section{Supplementary Information}

The online version contains supplementary material available at https://doi. org/10.1186/s12879-021-05879-1.

Additional file 1. Information sheet and consent forms.

\section{Abbreviations}

ACT: Artemisinin-based combination therapy; AE: Adverse event; Al: Active ingredient; CRF: Case report form; DHS: Demographic health survey; DSMB: Data safety and monitoring committee; EIR: Entomological inoculation rate; GPRIM: Global Plan for Insecticide Resistance Management 
in malaria vectors; HLC: Human landing catches; IEC: Information, education and communication; ITN: Insecticide-treated net; LLIN: Long-lasting insecticidal net; LSHTM: London school of hygiene and tropical medicine; MPAC: Malaria policy advisory group; MFO: Mixed function oxidases; NMCP: National malaria control programme; ODK: Open data kit; PCR: Polymerase chain reaction; PBO: Piperonyl butoxide; Pf-CSP: Plasmodium falciparum circumsporozoite protein; QC: Quality control; RCT: Randomized clinical trial; RDT: Rapid diagnostic test; SAE: Serious adverse event; SOP: Standard operating procedure; SPIRIT: Standard protocol items recommendations for interventional trials; SSA: Sub-Saharan Africa; TSC: Trial steering committee; VCAG: Vector Control Advisory Group; WHO: World health organization

\section{Acknowledgments}

The authors gratefully acknowledge the Trial Steering committee and Data Safety and Monitoring Committee members for safeguarding the interests of trial participants, assessing the safety and efficacy of the interventions during the trial, and monitoring the overall conduct of the trial. We also thank the local medical staff and community leaders of the Zou region for supporting the trial and for agreeing to collaborate with the study investigators. Electronic data solutions were provided by LSHTM Open Research Kits (odk.Ishtm.ac.uk).

\section{Authors' contributions}

$J C, C N, I K, L A M, M C A, M R$, and NP contributed to the development of this protocol. MA, JC, and NP drafted the protocol manuscript. MA, JC, CN, AS, $L A M, I K, M R, M C A$, and NP are responsible for the trial design, sample size calculations, and designing the analysis plan. MA, JC, ED, HA, CT, MCA, and $N P$ contributed to the data management and clinical monitoring plan. MA, $J C, C N, A S, E D, B Y, G G P, L A M, M R, M C A$, and NP designed the entomological and resistance monitoring plan. $M A, J C, C N, A S, E D, B Y, H A, A H, F T, M C A$, and NP participated in the net distribution plan. The final manuscript was read and approved by all authors.

\section{Funding}

This research is supported by a grant to the London School of Hygiene and Tropical Medicine from UNITAID and Global Fund via the Innovative Vector Control Consortium (IVCC). This cluster-randomized clinical trial is part of a larger project "The New Net project". The funders do not play a role in study design, collection, management, analysis and interpretation of data. They do not influence the writing of the report and the decision to submit.

\section{Availability of data and materials}

The full protocol is available from the study principal investigator (Dr. Natacha Protopopoff; Email: natacha.protopopoff@lshtm.ac.uk) upon reasonable request.

\section{Ethics approval and consent to participate}

This study is to be conducted following the principles outlined in the $\mathrm{ICH}$ Harmonized Tripartite Guideline for Good Clinical Practice and the Declaration of Helsinki. The Benin Ministry of Health ethics committee (Reference $\mathrm{N}^{\circ} 6 / 30 / \mathrm{MS} / \mathrm{DC} / \mathrm{SGM} / \mathrm{DRFMT/CNERS/SA)}$ ) approved this study. It is also approved by the institutional review board of the London School of Hygiene and Tropical Medicine (Reference $N^{\circ} 16237$ ) and the WHO Research Ethics Review Committee (Reference ERC.0003153).

Before any project activities, villages and hamlet leaders and local health staff will be invited to sensitisation sessions. Community health workers within each cluster will be fully informed as to the aims of the trial and will be on hand to answer day to day questions regarding the study. For all activities involving a household, written informed consent will be obtained from an adult guardian in the household or be given by the participant if over 18 years. All participation is completely voluntary, and participants can withdraw at any time. In case the participant does not understand French, study investigators will be trained to practice oral translation of the consent into local languages. Participants will be asked to sign the consent in duplicate, one will be kept by the project and the other will remain with the household. If the person consenting is unable to read or write, their fingerprint will be taken, and an impartial witness to the informed consent procedures signature will be requested to sign. For the active follow-up cohort, consent will be sought once at the first enrolment visit and will be performed by the study nurses. Assent will be sought for children over 10 years.
For HLCs, written informed consent will also be obtained from volunteer mosquito collectors before being involved in the study. Mosquito collectors will be over the age of 18 years. To prevent yellow fever, they will be vaccinated. Their health will be monitored by the project clinical team and free treatment for malaria will be provided, if necessary. Each volunteers will work one night every 3 months to reduce the risk.

\section{Consent for publication}

Not applicable.

\section{Competing interests}

The authors declare that they have no competing interests.

\section{Author details}

${ }^{1}$ Faculty of Infectious and Tropical Diseases, Disease Control Department, London School of Hygiene and Tropical Medicine, WC1E 7HT, London, UK. ${ }^{2}$ Medical Research Council (MRC) International Statistics and Epidemiology Epidemiology Group, London School of Hygiene and Tropical Medicine, WC1E 7HT, London, UK. ${ }^{3}$ Centre de Recherche Entomologique de Cotonou (CREC), Cotonou, Benin. ${ }^{4}$ Faculty of Agronomy, University of Parakou, Parakou, Benin. ${ }^{5}$ National Malaria Control Program, Ministry of Health, Cotonou, Benin. ${ }^{6}$ School of Epidemiology and Public Health, Faculty of Medicine, University of Ottawa, Ottawa, Canada. ${ }^{7}$ School of Pathology, Faculty of Health Sciences, University of Witwatersrand, Johannesburg, South Africa. ${ }^{8}$ Southern African Development Community Malaria Elimination Eight Secretariat, Windhoek, Namibia.

Received: 18 January 2021 Accepted: 9 February 2021

\section{Published online: 19 February 2021}

\section{References}

1. Tizifa TA, Kabaghe AN, McCann RS, van den Berg H, Van Vugt M, Phiri KS. Prevention efforts for malaria. Curr Trop Med Rep. 2018;5:41-50.

2. World Health Organization. World malaria report 2019. Geneva: World Health Organization; 2019. License: CC BY-NC-SA 3.0 IGO. 2019

3. Ranson H, N'guessan R, Lines J, Moiroux N, Nkuni Z, Corbel V. Pyrethroid resistance in African anopheline mosquitoes: what are the implications for malaria control? Trends Parasitol. 2011;27:91-8.

4. Asidi A, N'Guessan R, Akogbeto M, Curtis C, Rowland M. Loss of household protection from use of insecticide-treated nets against pyrethroid-resistant mosquitoes, Benin. Emerg Infect Dis. 2012;18:1101-6.

5. Ochomo EO, Bayoh NM, Walker ED, Abongo BO, Ombok MO, Ouma C, et al. The efficacy of long-lasting nets with declining physical integrity may be compromised in areas with high levels of pyrethroid resistance. Malar J. 2013;12:368.

6. Kleinschmidt I, Bradley J, Knox TB, Mnzava AP, Kafy HT, Mbogo C, et al. Implications of insecticide resistance for malaria vector control with longlasting insecticidal nets: a WHO-coordinated, prospective, international, observational cohort study. Lancet Infect Dis. 2018;18:640-9.

7. Tokponnon FT, Sissinto Y, Ogouyémi AH, Adéothy AA, Adechoubou A, Houansou T, et al. Implications of insecticide resistance for malaria vector control with long-lasting insecticidal nets: evidence from health facility data from Benin. Malar J. 2019;18:37.

8. World Health Organization. Global plan for insecticide resistance management in malaria vectors; 2012. p. 132.

9. Protopopoff N, Mosha JF, Lukole E, Charlwood JD, Wright A, Mwalimu CD, et al. Effectiveness of a long-lasting piperonyl butoxide-treated insecticidal net and indoor residual spray interventions, separately and together, against malaria transmitted by pyrethroid-resistant mosquitoes: a cluster, randomised controlled, two-by-two factorial design trial. Lancet Lond Engl. 2018;391:1577-88.

10. Staedke SG, Gonahasa S, Dorsey G, Kamya MR, Maiteki-Sebuguzi C, Lynd A, et al. Effect of long-lasting insecticidal nets with and without piperonyl butoxide on malaria indicators in Uganda (LLINEUP): a pragmatic, clusterrandomised trial embedded in a national LLIN distribution campaign. Lancet Lond Engl. 2020;395:1292-303.

11. Ngufor C, N'Guessan R, Fagbohoun J, Todjinou D, Odjo A, Malone D, et al. Efficacy of the Olyset Duo net against insecticide-resistant mosquito vectors of malaria. Sci Transl Med. 2016;8:356ra121.

12. Tiono AB, Ouédraogo A, Ouattara D, Bougouma EC, Coulibaly S, Diarra A, et al. Efficacy of Olyset duo, a bednet containing pyriproxyfen and 
permethrin, versus a permethrin-only net against clinical malaria in an area with highly pyrethroid-resistant vectors in rural Burkina Faso: a clusterrandomised controlled trial. Lancet Lond Engl. 2018;392:569-80.

13. Djènontin A, Ahoua Alou LP, Koffi A, Zogo B, Duarte E, N'Guessan R, et al. Insecticidal and sterilizing effect of Olyset Duo ${ }^{\oplus}$, a permethrin and pyriproxyfen mixture net against pyrethroid-susceptible and -resistant strains of Anopheles gambiae s.s.: a release-recapture assay in experimental huts. Parasite Paris Fr. 2015;22:27.

14. Ngufor C, Agbevo A, Fagbohoun J, Fongnikin A, Rowland M. Efficacy of Royal Guard, a new alpha-cypermethrin and pyriproxyfen treated mosquito net, against pyrethroid-resistant malaria vectors. Sci Rep. 2020;10:12227.

15. N'Guessan R, Odjo A, Ngufor C, Malone D, Rowland M. A Chlorfenapyr mixture net interceptor ${ }^{\circledast} \mathrm{G} 2$ shows high efficacy and wash durability against resistant mosquitoes in West Africa. PLoS One. 2016;11:e0165925.

16. Bayili K, N'do S, Namountougou M, Sanou R, Ouattara A, Dabiré RK, et al. Evaluation of efficacy of Interceptor ${ }^{\circledast} \mathrm{G} 2$, a long-lasting insecticide net coated with a mixture of chlorfenapyr and alpha-cypermethrin, against pyrethroidresistant Anopheles gambiae s.l. in Burkina Faso. Malar J. 2017;16:190.

17. Camara S, Ahoua Alou LP, Koffi AA, Clegban YCM, Kabran J-P, Koffi FM, et al. Efficacy of Interceptor ${ }^{\circledast} \mathrm{G} 2$, a new long-lasting insecticidal net against wild pyrethroid-resistant Anopheles gambiae s.s. from Côte d'Ivoire: a semi-field trial. Parasite Paris Fr. 2018;25:42.

18. Protopopoff N, Rowland M. Accelerating the evidence for new classes of long-lasting insecticide-treated nets. Lancet. 2018;391:2415-6.

19. Killeen GF, Ranson $\mathrm{H}$. Insecticide-resistant malaria vectors must be tackled. Lancet Lond Engl. 2018;391:1551-2.

20. World Health Organization. Design of epidemiological trials for vector control products : Report of a WHO expert advisory group. Geneva, Switzerland: WHO; 2017.

21. Hancock PA, Hendriks CJM, Tangena J-A, Gibson H, Hemingway J, Coleman $M$, et al. Mapping trends in insecticide resistance phenotypes in African malaria vectors. PLoS Biol. 2020;18:e3000633.

22. World Health Organization. Guidelines for laboratory and field-testing of long-lasting insecticidal nets (WHO/HTM/NTD/WHOPES/2013.1). Geneva: WHO; 2013.

23. Churcher TS, Lissenden N, Griffin JT, Worrall E, Ranson H. The impact of pyrethroid resistance on the efficacy and effectiveness of bednets for malaria control in Africa. eLife. 2016;5:e16090.

24. Sherrard-Smith E, Griffin JT, Winskill P, Corbel V, Pennetier C, Djénontin A, et al. Systematic review of indoor residual spray efficacy and effectiveness against plasmodium falciparum in Africa. Nat Commun. 2018;9:4982.

25. Chan A-W, Tetzlaff JM, Altman DG, Laupacis A, Gøtzsche PC, Krleža-Jerić K, et al. SPIRIT 2013 statement: defining standard protocol items for clinical trials. Ann Intern Med. 2013;158:200-7.

26. National Institute of Statistic and Economic Analysis (INSAE). National census, villages from Department of Zou (RGPH-4, 2013), Cotonou, Benin. 2016:83. Available: https://insae.bj/images/docs/insae-statistiques/demogra phiques/population/Effectifs\%20de\%20la\%20population\%20des\%20villa ges\%20et\%20quartiers\%20de\%20ville\%20du\%20benin/Cahier\%20Village\%2 ORGPH4\%202013.pdf. Accessed 11 Feb 2021.

27. National System of Health Information and Management (SNIGS)). National health statistics 2019, Cotonou, Benin, Ministry of Health. 2020:243. Available: https://sante.gouv.bj/assets/ressources/pdf/Annuaire\%20Sta tistique\%202019-1.pdf Accessed 11 Feb 2021.

28. National Institute of Statistic and Economic Analysis (INSAE). Demographic Health Survey in Benin, 2011-2012: Key indicators. Cotonou, Benin and Rockville, Maryland, USA: INSAE et ICF; 2013.

29. National Institute of Statistic and Economic Analysis (INSAE). Demographic Health Survey in Benin, 2017-2018: Key indicators. Cotonou, Bénin et Rockville, Maryland, USA: INSAE et ICF; 2019.

30. Ngufor C, N'Guessan R, Fagbohoun J, Subramaniam K, Odjo A, Fongnikin A, et al. Insecticide resistance profile of Anopheles gambiae from a phase II field station in Cové, southern Benin: implications for the evaluation of novel vector control products. Malar J. 2015;14:464.

31. World Health Organization. List of WHO prequalified Vector Control Products (WHO, Geneva, 2020). 2020.

32. Black BC, Hollingworth RM, Ahammadsahib KI, Kukel CD, Donovan S. Insecticidal action and mitochondrial uncoupling activity of AC-303,630 and related halogenated Pyrroles. Pestic Biochem Physiol. 1994;50:115-28.

33. Oxborough RM, N'Guessan R, Jones R, Kitau J, Ngufor C, Malone D, et al. The activity of the pyrrole insecticide chlorfenapyr in mosquito bioassay: towards a more rational testing and screening of non-neurotoxic insecticides for malaria vector control. Malar J. 2015;14. https://doi.org/10.11 86/s12936-015-0639-x.

34. Harris C, Lwetoijera DW, Dongus S, Matowo NS, Lorenz LM, Devine GJ, et al. Sterilising effects of pyriproxyfen on Anopheles arabiensis and its potential use in malaria control. Parasit Vectors. 2013:6:144.

35. World Health Organization. Report of the 20th WHOPES Working group meeting. Geneva: WHO; 2017. p. 4-46.

36. Seyoum D, Speybroeck N, Duchateau L, Brandt P, Rosas-Aguirre A. Longlasting insecticide net ownership, access and use in Southwest Ethiopia: a community-based cross-sectional study. Int J Environ Res Public Health. 2017;14(11):1312.

37. Mboma ZM, Overgaard HJ, Moore S, Bradley J, Moore J, Massue DJ, et al. Mosquito net coverage in years between mass distributions: a case study of Tanzania, 2013. Malar J. 2018;17:100.

38. Yunta C, Grisales N, Nász S, Hemmings K, Pignatelli P, Voice M, et al. Pyriproxyfen is metabolized by P450s associated with pyrethroid resistance in an. Gambiae. Insect Biochem Mol Biol. 2016;78:50-7.

39. Hayes RJ, Bennett S. Simple sample size calculation for cluster-randomized trials. Int J Epidemiol. 1999;28:319-26.

40. Ogouyemi-Hounto A, Kinde-Gazard D, Nahum A, Abdillahi A, Massougbodji A. Management of malaria in Benin: evaluation of the practices of healthcare professionals following the introduction of artemisinin derivatives. Med Trop Rev Corps Sante Colon. 2009;69:561-4.

41. Gillies MT, De Meillon B. The Anophelinae of Africa south of the Sahara (Ethiopian zoogeographical region). Publ South Afr Inst Med Res. 1968:54:1-343.

42. Detinova TS, Gillies MT. Observations on the determination of the age composition and epidemiological importance of populations of Anopheles gambiae Giles and Anopheles funestus Giles in Tanganyika. Bull World Health Organ. 1964;30:23-8.

43. Wirtz RA, Duncan JF, Njelesani EK, Schneider I, Brown AE, Oster CN, et al. ELISA method for detecting plasmodium falciparum circumsporozoite antibody. Bull World Health Organ. 1989;67:535-42.

44. Scott JA, Brogdon WG, Collins FH. Identification of single specimens of the Anopheles gambiae complex by the polymerase chain reaction. Am J Trop Med Hyg. 1993:49:520-9.

45. World Health Organization. Test procedures for insecticide resistance monitoring in malaria vector mosquitoes. Geneva, Switzerland: World Health Organisation; 2016.

46. Vatandoost H, Abai MR, Akbari M, Raeisi A, Yousefi H, Sheikhi S, et al. Comparison of CDC bottle bioassay with WHO standard method for assessment susceptibility level of malaria vector, Anopheles stephensi to three Imagicides. J Arthropod-Borne Dis. 2019;13:17-26.

47. Christophers S. The development of the egg follicle in Anophelines. Paludism. 1911;2:73-8.

48. Mavridis K, Wipf N, Medves S, Erquiaga I, Müller P, Vontas J. Rapid multiplex gene expression assays for monitoring metabolic resistance in the major malaria vector Anopheles gambiae. Parasit Vectors. 2019;12:9.

49. Council for International Organizations of Medical Sciences. International Ethical Guidelines for Biomedical Research Involving Human Subjects. Geneva, Switzerland: World Health Organization; 2002.

50. World Health Organization. Conditions for deployment of mosquito nets treated with a pyrethroid and piperonyl butoxide, G.M. Programme, Editor. World Health Organization: Geneva 2017.

51. Koffi AA, Ahoua Alou LP, Djenontin A, Kabran J-PK, Dosso Y, Kone A, et al. Efficacy of Olyset $^{\circledR}$ Duo, a permethrin and pyriproxyfen mixture net against wild pyrethroid-resistant Anopheles gambiae s.s. from Côte d'Ivoire: an experimental hut trial. Parasite Paris Fr. 2015;22:28.

52. Toé KH, Mechan F, Tangena J-AA, Morris M, Solino J, Tchicaya EFS, et al. Assessing the impact of the addition of pyriproxyfen on the durability of permethrin-treated bed nets in Burkina Faso: a compound-randomized controlled trial. Malar J. 2019;18:383.

53. World Health Organization. Data requirements and protocol for determining noninferiority of insecticide-treated net and indoor residual spraying products within an established WHO intervention class (WHO/CDS/GMP/2018.22.Rev.1.). 2019.

54. Vinit R, Timinao L, Bubun N, Katusele M, Robinson LJ, Kaman P, et al. Decreased bioefficacy of long-lasting insecticidal nets and the resurgence of malaria in Papua New Guinea. Nat Commun. 2020;11:3646.

\section{Publisher's Note}

Springer Nature remains neutral with regard to jurisdictional claims in published maps and institutional affiliations. 